\title{
Orbital-selective Mottness in layered iron oxychalcogenides: The case of $\mathrm{Na}_{2} \mathrm{Fe}_{2} \mathrm{OSe}_{2}$
}

\author{
L. Craco, ${ }^{1}$ M.S. Laad, ${ }^{2}$ and S. Leoni ${ }^{3}$ \\ ${ }^{1}$ Instituto de Física, Universidade Federal de Mato Grosso, 78060-900, Cuiabá, MT, Brazil \\ ${ }^{2}$ Institut Laue-Langevin, 6 Rue Jules Horowitz, 38042 Grenoble Cedex, France \\ ${ }^{3}$ Physical Chemistry, Technical University Dresden, 01062 Dresden, Germany
}

(Dated: October 1, 2018)

\begin{abstract}
Using a combination of local-density approximation (LDA) and dynamical mean-field theory (DMFT) calculations, we explore the correlated electronic structure of a member of the layered iron oxychalcogenide $\mathrm{Na}_{2} \mathrm{Fe}_{2} \mathrm{OSe}_{2}$. We find that the parent compound is a multi-orbital Mott insulator. Surprisingly, and somewhat reminiscent of underdoped high- $T_{c}$ cuprates, carrier localization is found to persist upon hole doping because the chemical potential lies in a gap structure with almost vanishing density of states. On the other hand, in remarkable contrast, electron-doping drives an orbital-selective metallic phase (OSMP) with coexisting pseudogaped (Mott-localised) and itinerant carriers. These remarkably contrasting behaviors in a single system thus stem from drastic electronic reconstruction caused by large-scale transfer of dynamical spectral weight involving states with distinct orbital character at low energies, putting the oxychalcogenides neatly into the increasingly visible tendency of Fe-based systems as ones in OSM phases. We detail the implications that follow from our analysis, and discuss the nature and symmetries of the superconductive states that may arise upon proper doping or pressurising $\mathrm{Na}_{2} \mathrm{Fe}_{2} \mathrm{OSe}_{2}$.
\end{abstract}

\section{INTRODUCTION}

Recent finding of unconventional high- $\mathrm{T}_{c}$ superconductivity (HTSC) in Fe arsenides and selenides have reinvigorated the HTSC revolution. In particular, the outstanding and vigorous debates concerning the normal state giving way to HTSC in cuprates have also produced their mark upon the discussion of mechanism(s) of HTSC in Fe-based superconductors (FeSC): Does HTSC result as a pairing instability of a conventional Landau-Fermi liquid (LFL),$\frac{1}{,}$ or as one of a non-LFL, akin to the cuprates $\stackrel{2}{2}$ Clearly, one possible way to resolve this very important issue is to investigate as many members of the Fe-based family as possible.

To date, both, normal paramagnetic and antiferromagnetically ordered phases in various members have been extensively studied. It has slowly emerged that, in their normal, paramagnetic state the FeSC (both pnictides and chalcogenides) increasingly fall into the bad metal category. Usually, in correlated systems, the generic picture is known by now to be one where a high- $T$ bad-metal undergoes a $T$-dependent crossover to a correlated or heavy LFL at low temperature $(T)$. This is due to the development of a severely renormalised lattice coherence scale, driven by increasing relevance of electronic correlations as $T$ reduces. We will refer to this behavior as "standard" for correlated metals, and thus any fundamental deviation from this generic behavior will be termed "anomalous". According to this definition, increasing number of Fe-based compounds fall into the anomalous class, in the sense that LFL behavior is seemingly not recovered at low $T$ when superconductivity (SC) is suppressed by appropriate perturbations. In $\mathrm{SmFeAsO}_{1-x} \mathrm{~F}_{x} \stackrel{3}{,}$ for example, destruction of $\mathrm{SC}$ by high magnetic fields reveals a low- $T$ insulator-like state as in high- $T_{c}$ cuprates. 4 $\mathrm{NdFeAsO}_{1-x} \mathrm{~F}_{x}$ shows similar behavior upon irradiationinduced disorder. $\frac{5}{=}$ In both cases, the residual resistivity,
$\rho_{r}(T) \simeq-\log T$, suggesting an anomalous field- or radation (disorder) driven bad-metal-insulator transition atlow $T$ in the normal state, and that the normal state might thus not be as normal as thought. SC even arises directly from an even more incoherent semiconductorlike normal state in $\mathrm{FeSe}_{1-x} \mathrm{Te}_{x}, \underline{6}$ Optical and spectroscopic studies in 122-iron selenide ${ }^{7}$ superconductors show large-scale spectral weight transfer (SWT) as a function of temperature across the magnetic and superconducting instabilities, a fingerprint of Mottness $\underline{8}$ Both $\rho(T) \simeq T$ and bad metallicity above $T_{c}$ or the Néel temperature $\left(T_{N}\right)$ are features shared along with other non-LFL metals close to a Mott instability: $\frac{9}{}$ Thus, the non-LFL metallic state in the above cases strongly points toward a fundamental role for Mott localization. ${ }^{10}-12$

In this context, recent finding of insulating, $\mathrm{AF}$ ground state in layered Fe-oxychalcogenides ${ }^{12}-16$ is interesting, since it confirms theoretical suggestions that Mott-insulating parent compounds in the FeSC systems could be found by increasing the ratio of the interactionto-hopping $(U / W)$ beyond a critical value for a Mott transition. 11,17 Evidence for Fe-layered compounds with different intergrowth structures is very interesting: Its generic effect is to reduce the one-electron bandwidths 12 $(W)$ by employing two-dimensional secondary building units, making it possible to realize almost isolated two-dimensional Fe-layers. The newly discovered Feoxychalcogenides thus help to sharpen the fundamental debate ${ }^{18}$ on the degree of electronic correlations in FeSC in general. Finding of large local moment value on $\mathrm{Fe}, \mathbf{M}_{F e} \simeq 2.23 \mu_{B},{ }^{14}$ along with large activation energies $^{12-15}$ suggests strong electronic localization. The consequences of Mottness upon physical responses of oxychalcogenides has, however, not received the attention it deserves.

Here we adopt a Mottness view with incorporation of sizable multi-orbital (MO) correlations in the Fe $d$ shell. 

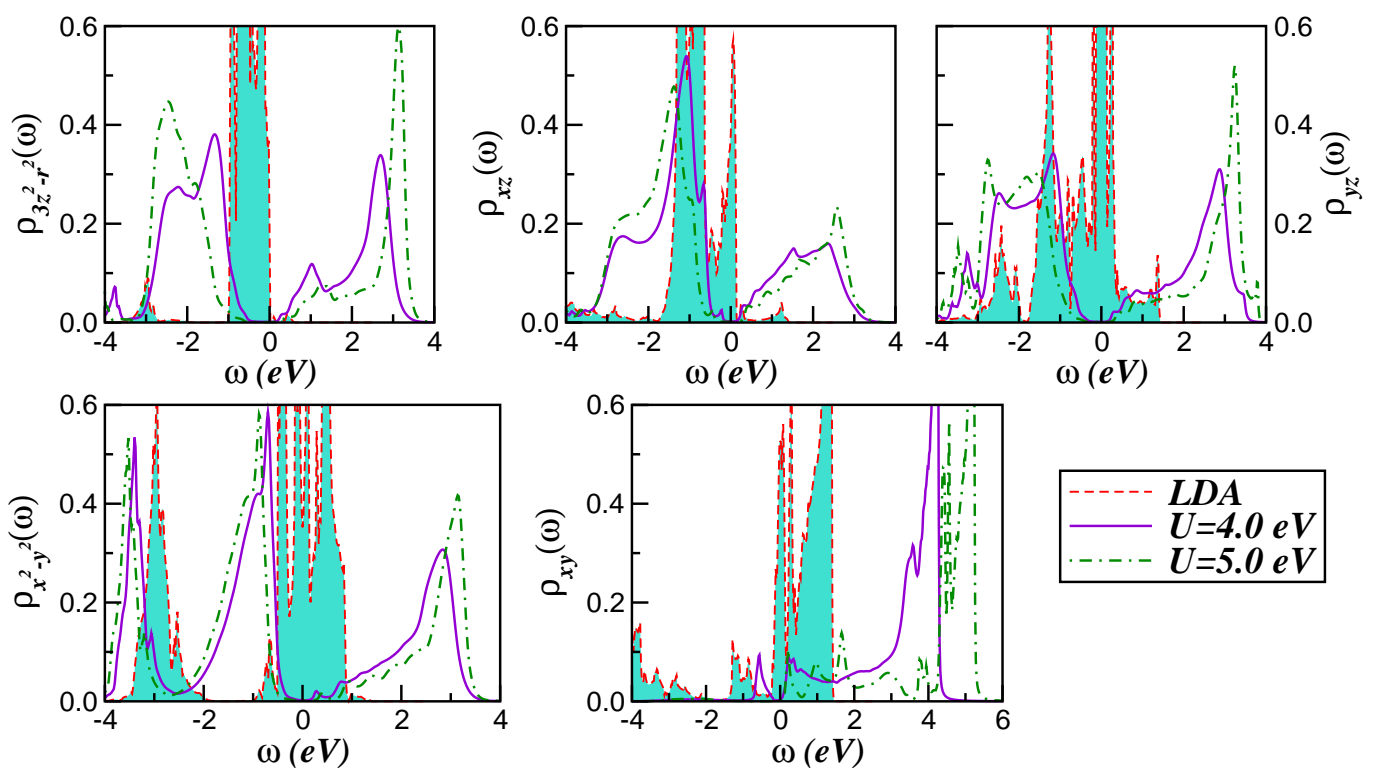

FIG. 1: (Color online) Orbital-resolved LDA and LDA+DMFT (two values of $U$ and fixed $J_{H}=0.7 \mathrm{eV}$ ) density-of-states (DOS) for the Fe $d$ orbitals of $\mathrm{Na}_{2} \mathrm{Fe}_{2} \mathrm{OSe}_{2}$. Notice the narrow bands in the LDA DOS. Compared to the LDA results, large spectral weight transfer along with Mott localization is visible in the LDA+DMFT spectral functions.

As shown below, all five $d$-bands must be kept at a minimally realistic level in order to satisfactorily resolve the Mott insulating and bad metallic regimes obtained, respectively, in pure and doped compounds. In Mott insulators, sizable electronic correlations drives new physical effects upon (electron, hole) doping. They can induce a pseudogap regime referred to above, where the chemical potential lies in an energy region of vanishing density of states (DOS) $\stackrel{19}{\Perp}$ or orbital-selective (OS) incoherent states, naturally yielding co-existent insulating and bad metallic states as in cuprates or in increasing number of pnictides $\stackrel{20-22}{\underline{2}}$ In this work, we use the localdensity approximation plus dynamical mean-field theory $(\mathrm{LDA}+\mathrm{DMFT})^{23}$ to study these issues. We also use these results to discuss the influence of Mottness on issues mentioned above in lightly doped $\mathrm{Na}_{2} \mathrm{Fe}_{2} \mathrm{OSe}_{2}$ and follow this up with specific predictions which can be tested in future experiments.

\section{RESULTS AND DISCUSSION}

The crystal structure of $\mathrm{Na}_{2} \mathrm{Fe}_{2} \mathrm{OSe}_{2}$ (space group $I 4 / \mathrm{mmm}$ ) is built from alternate stacking of $\mathrm{Fe}_{2} \mathrm{OSe}_{2}$ blocks and double layers of $\mathrm{Na}$ along c-axis. In the $\mathrm{Fe}_{2} \mathrm{OSe}_{2}$ unit, $\mathrm{Fe}^{2+}\left(d^{6}\right.$ configuration $)$ ion is located between oxygen atoms, forming a square-planar layer, which is an anti-configuration to with respect to the $\mathrm{CuO}_{2}$ layer of high- $T_{c}$ cuprates. Using the cell parameter values $\stackrel{15}{\stackrel{15}{t}}$ we performed LDA calculations for $\mathrm{Na}_{2} \mathrm{Fe}_{2} \mathrm{OSe}_{2}$ within the linear muffin-tin orbitals (LMTO) scheme, $\stackrel{24}{\stackrel{2}{4}}$ in the atomic sphere approximation. Self-consistency was reached by performing calculations with 163 irreducible k-points. The radii of the atomic spheres were chosen as $r=2.512(\mathrm{Fe}), r=3.05(\mathrm{Na}), r=3.422(\mathrm{Se})$ and $r=1.99$ (O) a.u. in order to minimize their overlap. Within LDA, the one-electron part of the Hamiltonian reads $H_{0}=\sum_{\mathbf{k}, a, \sigma} \epsilon_{a}(\mathbf{k}) c_{\mathbf{k}, a, \sigma}^{\dagger} c_{\mathbf{k}, a, \sigma}$, where $a=\left(3 z^{2}-\right.$ $\left.r^{2}, x z, y z, x^{2}-y^{2}, y z\right)$ label the (diagonalized in orbital basis) five Fe $3 d$ bands, which are the only ones we retain, since the non- $d$-orbital DOS have negligible or no weight at $E_{F}$.

\section{A. Electronic structure}

In Fig. 1 we show the LDA one-electron band structure, confirming that the active electronic states involve the $\mathrm{Fe}$ $d$ carriers. A sizable reduction $(\mathrm{O}(30) \%)$ of the average LDA bandwidth $\left(W_{L D A}\right)$ relative to that of tetragonal FeSe,, 11 induced by hybridisation with oxygen atoms and distorting effects of the extra Na layers, is obtained. As shown previously, FeSe is already a bad metal close to a Mott insulator, 11 and the significantly smaller $W_{L D A}$ for $\mathrm{Na}_{2} \mathrm{Fe}_{2} \mathrm{OSe}_{2}$ then naturally implies favoring a Mott insulator in oxychalcogenides. The MO-driven anisotropies in the LDA band structure are also manifested in Fig. 1. As is common to all FeSC, the $3 z^{2}-r^{2}, x y$ orbitals exhibit the bonding/antibonding splitting characteristic of the tetragonal unit cell. These bands, almost totally bandgapped near the Fermi energy $\left(E_{F}\right)$ in the FeSC systems go over into highly orbitally polarized, narrow bands in $\mathrm{Na}_{2} \mathrm{Fe}_{2} \mathrm{OSe}_{2}$. A similar feature is found for the $x z$ orbital. 
Moreover, the real crystal-field splitting also severely renormalizes the LDA orbital occupancies (promoting enhanced orbital polarization) of the $3 z^{2}-r^{2}, x z, x y$ sectors as well. In another important distinction to the FeSC, the (strong) crystal-field splitting also lifts the degeneracy of the $x z, y z$ orbitals, leaving an AF ground state at low- $T$ without any tetragonal-to-orthorhombic structural phase transition. It thus follows that the novelties found in FeSCs, relating to electronic nematic instabilities in the tetragonal phase near the borderline of structural and magnetic transitions, will not play an active role in oxychalogenides. Thus, oxychalcogenides should show very different responses upon carrier doping: elucidating precisely this aspect with a well-controlled approach will be our aim in this work.

We now discuss our LDA+DMFT results obtained within the $d^{6}$ configuration of the $\mathrm{Fe}^{2+}$ in $\mathrm{Na}_{2} \mathrm{Fe}_{2} \mathrm{OSe}_{2}$. Starting with the five Fe $d$-orbitals in LDA, we use MODMFT $^{10}$ to derive the correlated $d$-band spectral functions. We use the MO iterated perturbation theory as an impurity solver for DMFT 25 This perturbative, manyparticle scheme has a proven record of recovering correct LFL behavior, and bad-metallicity in correlated oxides. In the MO problem: $H=H_{0}+H_{\text {int }}$ with

$$
\begin{aligned}
H_{\text {int }} & =U \sum_{i, a} n_{i, a \uparrow} n_{i, a \downarrow} \\
& +\sum_{i, a \neq b}\left[U^{\prime} n_{i, a} n_{i, b}-J_{H} \mathbf{S}_{i, a} \cdot \mathbf{S}_{i, b}\right],
\end{aligned}
$$

$U^{\prime} \equiv U-2 J_{H}\left[U\left(U^{\prime}\right)\right.$ is the intra- (inter-) orbital Coulomb repulsion] and $J_{H}$ is the Hund's rule coupling. ${ }^{23}$ We choose values of $U=4.0 \mathrm{eV}, J_{H}=0.7 \mathrm{eV}$ as employed in our earlier works $\frac{10,26}{1}$ Though not determined $a b$ initio our parameter choice is consistent with our earlier and other theoretical works and with our underlying view that Fe-pnictides and -chalcogenides are better regarded as sizably correlated metals ${ }^{26,27}$

\section{B. Mott-insulating phase}

In the case of undoped $\mathrm{Na}_{2} \mathrm{Fe}_{2} \mathrm{OSe}_{2}$, our LDA+DMFT results, shown in Fig. 1 exhibit a clear Mott insulating gap in the one-particle spectral function. Several interesting features are clearly stand out: (i) The Mott gap is orbital-dependent, i.e, intrinsically anisotropic. (ii) Examination of the orbital-resolved spectral functions reveal a behavior hitherto known to FeSC systems. Namely, all orbitals are partially populated due to $U^{\prime}$ induced dynamical inter-orbital entanglement, 25 while the total orbital population is integral, as it should be for undoped $(\delta=0) \mathrm{Na}_{2} \mathrm{Fe}_{2} \mathrm{OSe}_{2}$. As seen in Fig. 1 , strong dynamical MO correlations originating from $U, U^{\prime}$ and $J_{H}$ lead to sizable spectral weight redistribution over large energy scales and the formation of a severely reconstructed (compared to LDA) correlated electronic structure. This feature is characteristic of multiband Mott systems, with concomitantly modified upper and lower Hubbard bands at high-energies: These latter features are related to the strongly coupled spin-orbital local moments defining a Mott insulator without long-range orbital or magnetic order. Though our results pertain to $T=0$, they are thus formally a valid description of the high- $T$ Mott insulator state described before.

\section{Filling-controlled electronic transition}

What happens upon carrier doping? Even though no data exists, the generic appearance of novel metallic states with low energy pseudogaps, and the instabilities of such states to unconventional order, and, in particular, to HTSC, in a wide variety of other correlated matter makes this a very important question to inquire about. As alluded to in the introduction, our aim here is to build upon the strengths of correlated electronic structure modelling to predict the effect of carrier doping. In particular, based on explicit calculations, we will present a set of predictions which could be tested in future experimental work.

In Fig. 2 we show the changes in the correlated electronic structure upon hole doping $(n \equiv 6+\delta$, with $\delta<0)$ the Mott insulator. An intriguing observation is that the Mott localisation-delocalisation phase transition does not occur at small doping. However, as $|\delta|$ increases, a selective-Mott state develops, in which the $x z, y z, x^{2}-y^{2}$ spectral functions show behavior of a Mott insulator with vanishing DOS at $E_{F}$, while the $3 z^{2}-r^{2}, x y$ orbitals now show (selective) metallic behavior, characterized by the presence of incoherent in-gap states at $E_{F}$. This is a clear demonstration of strong Mottness $\frac{19}{9}$ and an orbitalselective Mott transition (OSMT) in $\mathrm{Na}_{2} \mathrm{Fe}_{2} \mathrm{OSe}_{2}$. What is the origin of these features? In a MO system like the oxychalcogenides, strong (incoherent) scattering between different carriers in orbital states split relative to each other due to the specific crystal field (see discussion of this aspect above) leads to two main effects: (i) It leads, via static-Hartree contributions (from the static part of the orbital-dependent self-energies) to anorbital-dependent shifts of the $d$-bands relative to each other, and (ii) strong dynamical correlations due to sizable $U, U^{\prime}, J_{H}$ cause appreciable SWT over large energy scales, from high to low-energy, upon carrier doping. This second feature leads to a drastic modification of the spectral lineshapes. In full accord with our earlier qualitative discussion, the increase of the effective $U / W$ ratio in the present case relative to $\mathrm{FeSe}$ and $\mathrm{Fe}$-arsenides leads to increased low-energy incoherence, as the pronounced pseudogap and very broad low-energy spectral features clearly shows. Microscopically, strong incoherent scattering, arising from co-existence of Mott-localized and bad metallic states, leads to an almost complete suppression of the LFL quasiparticles and the emergence of an incoherent (pseudogaped) spectra, reminiscent of what is seen in cuprate oxides. Microscopically, infrared 

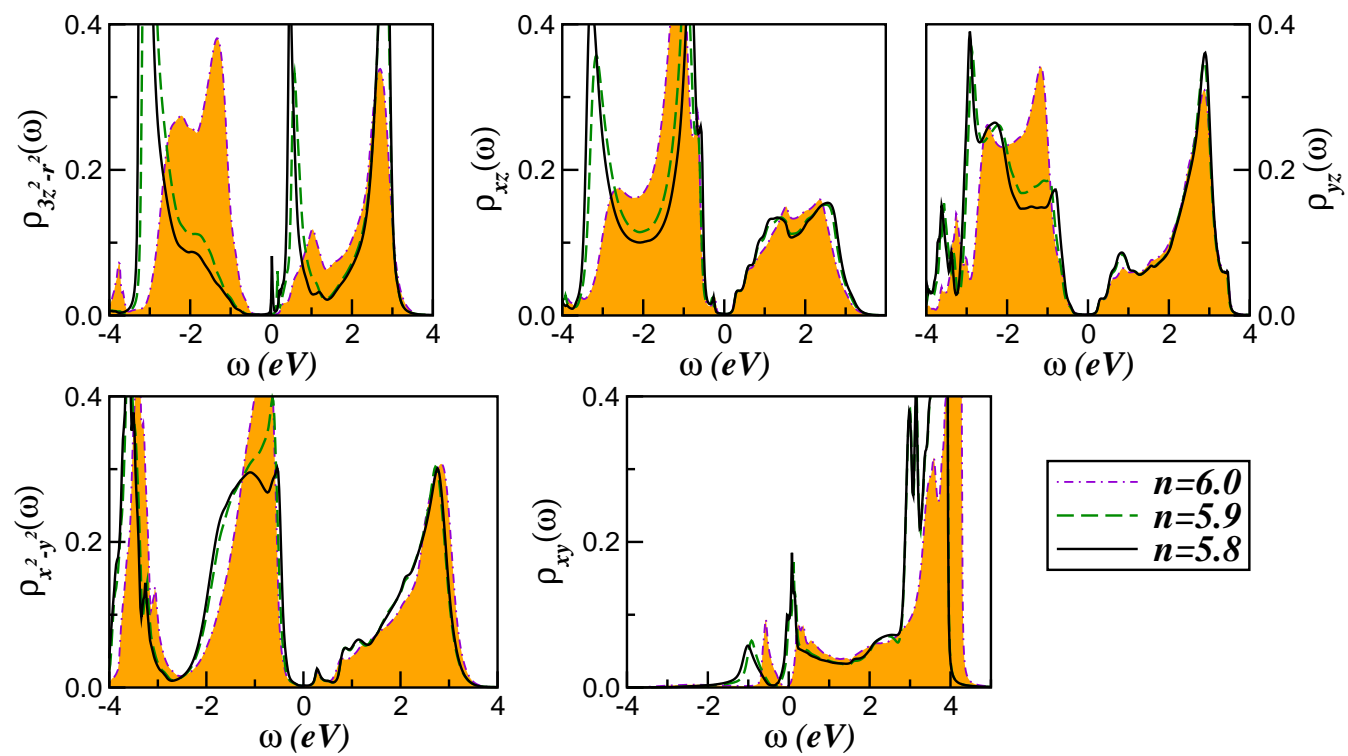

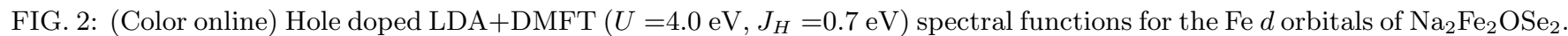
As in cuprates, ${ }^{19}$ strong hole localization is found in the lightly doped regime: only narrow gap states are formed in the slightly more delocalized $3 z^{2}-r^{2}$, xy orbitals.
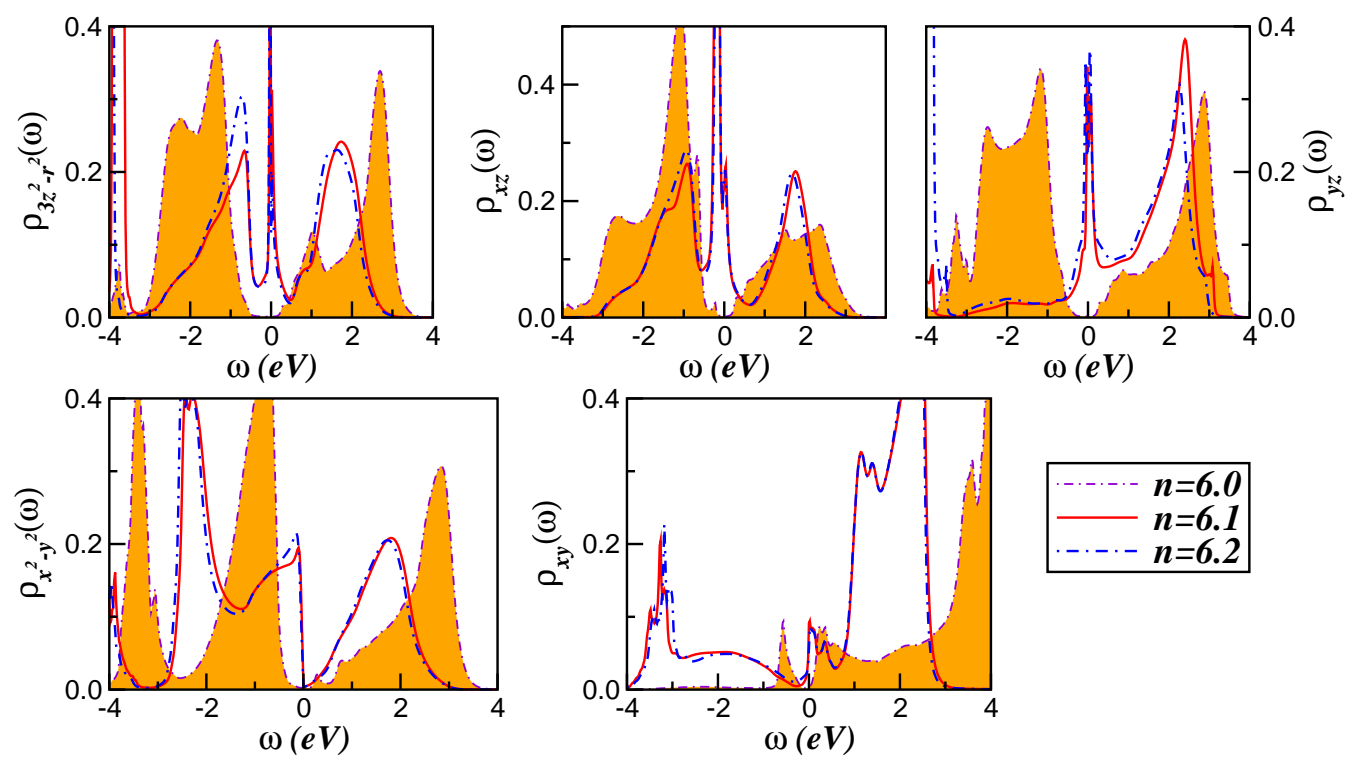

FIG. 3: (Color online) Effect of electron doping on the LDA+DMFT ( $U=4.0 \mathrm{eV}, J_{H}=0.7 \mathrm{eV}$ ) spectral functions for the Fe $d$ orbitals of $\mathrm{Na}_{2} \mathrm{Fe}_{2} \mathrm{OSe}_{2}$. Electron delocalization and appearance of narrow quasiparticle resonances near the Fermi energy is seen within the $3 z^{2}-r^{2}, x z, y z$ channels. Selective-localization and pseudogap features are the fingerprints of electronic correlations within the $x^{2}-y^{2}, x y$ orbitals.

LFL behavior (narrow Kondo resonance in DMFT) in the $3 z^{2}-r^{2}, x y$ orbitals is almost extinguished by strong scattering between the Mott-localized $x z, y z, x^{2}-y^{2}$ and the quasi-itinerant $3 z^{2}-r^{2}, x y$ components of the (DMFT) matrix-spectral function, due to sizable $U^{\prime}, J_{H}$, and is a clear manifestation of the OSMT in the five-band Hub- bard model we use.

Since there is no particle-hole symmetry in the system, it is interesting to inquire as to the effects of electron doping $(n \equiv 6+\delta, \delta>0)$ in $\mathrm{Na}_{2} \mathrm{Fe}_{2} \mathrm{OSe}_{2}$. In particular, we want to study if electron doping is qualitatively different; i.e, whether incoherent non-LFL behavior still 
survives in the infrared, or if coherent LFL response is favored. Fig. [3 exhibits the answer: The $x^{2}-y^{2}, x y$ spectral functions show clear pseudogap behavior, while, very interestingly and in stark contrast to the hole-doped case, the $3 z^{2}-r^{2}, x z, y z$ spectral functions now show narrow LFL-like quasiparticles at $E_{F}$. Hence, electron doped $\mathrm{Na}_{2} \mathrm{Fe}_{2} \mathrm{OSe}_{2}$ is predicted to lead to a more coherent (in the LFL sense in the infrared) state than its hole-doped counterpart above. The situation seems to be somewhat similar to the 122 -FeAs systems, $\stackrel{28}{=}$ where both coherent LFL-like and strongly incoherent non-LFL responses, dependent on the type and extent of doping, are seemingly observed. We point out, however, that such comparisons are inevitably fraught with danger, especially since the $x z, y z$ orbital degeneracy and the soft orbital fluctuations characteristic of the 122 -FeAs systems do not play a role in the Fe-oxychalcogenides.

The electron-hole asymmetry and selective-Mott transition in $\mathrm{Na}_{2} \mathrm{Fe}_{2} \mathrm{OSe}_{2}$ is further visible in orbital resolved self-energies, $\Sigma_{a \sigma}(\omega)$, shown in Fig. 4. Noticeable features include: (i) The insulating state is manifested by a sharp pole in $\operatorname{Im} \Sigma_{a \sigma}(\omega)$ as well by divergent $\operatorname{Re} \Sigma_{a \sigma}(\omega)$ (not shown) close to $E_{F}$. (ii) In the doped regime, only the higher (in energy) $x y$ orbital shows LFL $\left(\operatorname{Im} \Sigma_{a \sigma} \approx-\omega^{2}\right)$ form at small $\omega$. On the other hand, (iii) the imaginary part of the self-energy of the $3 z^{2}-r^{2}$ orbital at $E_{F}$ is finite (i.e., $\left.\operatorname{Im} \Sigma_{3 z^{2}-r^{2} \sigma}(\omega=0)<0\right)$. As is known, such behavior is caused by strong scattering between Mott localized and quasi-itinerant electronic states in the MO-DMFT problem, which maps onto an effective spinful Falicov-Kimball model in the local limit. These results imply that underdoped oxychalcogenides should be located in pseudogap regime and the metallic state obtained by the filling-controlled Mott transition is unconventional ${ }^{10}$ The origin of this unconventional metal is the lattice orthogonality catastrophe that occurs due to orbital-selective blocking of the coherent motion of the doped hole in the DMFT due to sizable $U^{\prime}, J_{H}$ in the MO Hubbard model.

Armed with the above results,,$\underline{26}$ we now attempt to predict the detailed physical response in the paramagnetic state of $\mathrm{Na}_{2} \mathrm{Fe}_{2} \mathrm{OSe}_{2}$. In Fig. [5, we show the total LDA+DMFT spectral functions. Clear Mott insulator features are visible for $\delta=0$, and we propose that future photoemission and X-ray absorption spectroscopy results, which probe one-electron subtraction and addition spectra, can be directly compared with these: In particular, a broad incoherent peak below $-0.8 \mathrm{eV}$ should be seen in both pure and doped cases. Additionally, drastic modification of the LDA+DMFT spectra with $\delta \neq 0$ is clearly visible. Our results for the hole- and electron-doped spectral functions $(n=5.8,6.2)$ reveal an clear differences, as found above: For hole-doped case, we find an almost totally incoherent spectral response due to the almost total blocking of coherent one-electron dynamics due to the lattice "orthogonality catastrophe" as noted above. For electron doping, however, a small quasicoherent LFL component is visible, notwithstanding
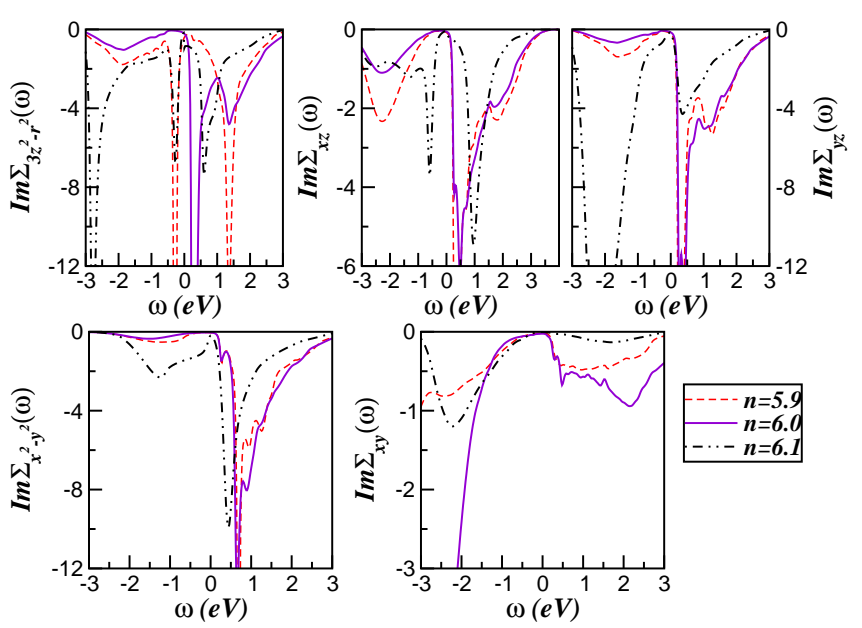

FIG. 4: (Color online) Orbital-resolved imaginary parts of the self-energies for the $\mathrm{Fe} d$ orbitals of stoichiometric and (electron/hole) doped $\mathrm{Na}_{2} \mathrm{Fe}_{2} \mathrm{OSe}_{2}$ with $U=4.0 \mathrm{eV}, J_{H}=0.7 \mathrm{eV}$. Notice the evolution of the sharp pole in the self-energies near $E_{F}$ across the doping-induced selective-Mott delocalization.

large-scale dynamical spectral weight transfer common in both cases. These are stringent tests for our proposal, and experimental verification should place it on solid ground. More distinguishing tests would be characteristic signatures in transport: For example, incoherent bad-metallicity with clear non-LFL $T$-dependence of the resistivity, $\rho(T) \simeq T^{n}$ with $0 \leq n<1.5$ should be seen in the hole-doped non-LFL cases, while much more conventional LFL-like resistivity, $\rho(T) \simeq T^{2}$ should be seen in the electron-doped cases.

One of our central results is the finding of co-existing orbital-selective incoherent and heavily dressed but coherent charge carriers. This behavior is reminiscent of what is found in the pseudogap regime in the underdoped HTSC cuprates. However, in cuprates, this differentiation of electronic states occurs in momentum space: Carriers in the nodal region are more quasicoherent than ones along the antinodal direction, which remain incoherent and in Mott localised states. It has been demonstrated, using a cluster-to-orbital mapping 29 that this nodal/antinodal differentiation in one-band Hubbard models is the momentum-space analog 30 of the orbitalselective Mott transition in models with several active orbitals. This is also borne out by our findings here, where the frequency dependence of the imaginary part of a subset (of orbitals self-energies) in Fig. 4 show Mott insulator features, while others show quasicoherent behavior. Viewed in terms of the above mapping, an analogy can be drawn with the observations of cellularDMFT studies, $\stackrel{23,30}{=}$ wherein similar features within the two-site cluster-DMFT were found: Suppressed coherence of antinodal quasiparticles originated from Mott physics while the nodal states remained protected against Mott localisation. Selective-localization in momentum 


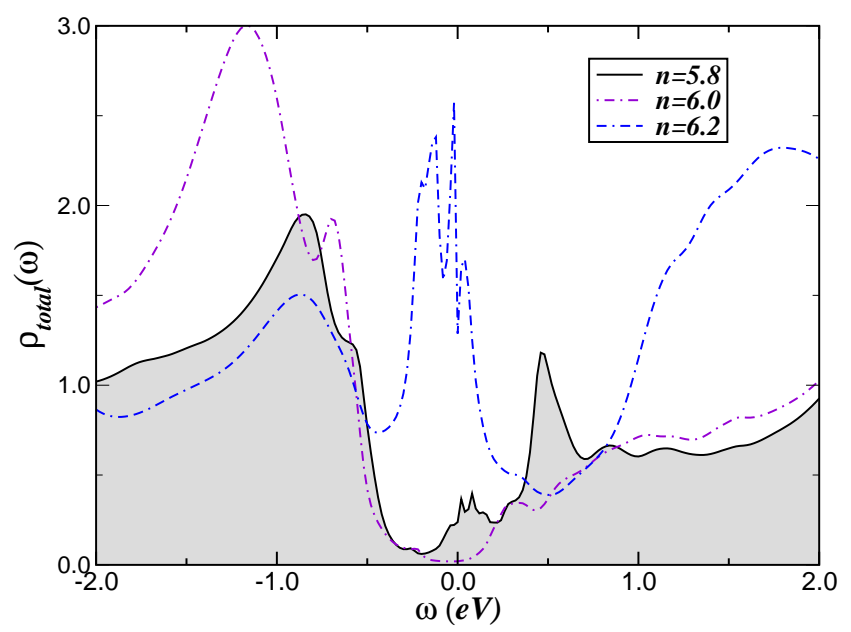

FIG. 5: (Color online) Total density-of-states for the Fe $d$ orbitals of stoichiometric and (electron/hole) doped $\mathrm{Na}_{2} \mathrm{Fe}_{2} \mathrm{OSe}_{2}$ with $U=4.0 \mathrm{eV}, J_{H}=0.7 \mathrm{eV}$. Notice the modification of the LDA+DMFT spectra from a Mott insulator $(n=6)$ to an almost totally incoherent electronic state $(n=5.8)$ due to large-scale transfer spectral weight. Selective microscopic coexistence of coherent-incoherent lowenergy electronic states is predicted for electron-doped $(n=$ 6.2) $\mathrm{Na}_{2} \mathrm{Fe}_{2} \mathrm{OSe}_{2}$.

(high- $T_{c}$ cuprates) or in orbital (FeSC) space thus seems to be a common element that provides a common, microscopic origin for the suppression of LFL coherence and emergence of non-LFL normal state properties of unconventional metals. $\underline{4}$

\section{Mechanisms of unconventional superconductivity}

Finally, motivated by a host of studies $1,31-33$ which propose the possibility and gap-function symmetry of superconducting states based on the nature (LFL or nonLFL) of the normal state and the nature of the renormalised Fermi surface(s), we attempt to carry out a qualitative program in our case. Specifically, we will focus on two normal state features: (i) The LFL or non-LFL character of the normal state(s) found above, and (ii) the natures of the renormalised Fermi surfaces to explore the range of superconduting state(s) that may result.

(i) Hole doping: Since LFL picture has been totally extinguished in the normal state for hole doping, it follows that the instability to any unconventional superconducting state cannot, by definition, proceed via the conventional route of BCS-like pairing of (heavily dressed or otherwise) long-lived quasiparticles: It is then much more likely, as occurs in critical metals, $\frac{32}{2}$ that SC pairing occurs as a result of the relevance of the inter-site, inter-orbital two-particle residual interactions in the incoherent metal. As worked out for the multiband situa- tion characteristic of Fe-based systems, such a residual interaction should involve pair-hopping electronic processes with MO character. In the hole-doped case above, with metallic $x y, 3 z^{2}-r^{2}$ states crossing $E_{F}$, we expect pairing to primarily involve solely the $x y$ band or both $x y, 3 z^{2}-r^{2}$ states if the Fermi surface has appreciable warping along $k_{z}$. Once $\mathrm{SC}$ has stabilised in the $x y$ band, an interband proximity effect characteristic of multiband $\mathrm{SC}^{34}$ must eventually take over to give full three-dimensional SC. Since the two-particle hopping is expected to acquire the frustrated form factor $\gamma(k)=\left(\cos k_{x}+\cos k_{y}\right)+\alpha \cos k_{x} \cos k_{y}$ (since the residual interaction scales as the second power of the one-electron hopping), 32 the SC gap function may or may not have inplane nodes, depending on whether the renormalised $x y$ band dispersion intersects the superconducting gap function.

(ii) Electron doping: Since more quasicoherent normal state behavior sets in for electron-doping, a more conventional analysis for superconducting pairing is mandated. Remarkably, since LFL-like behavior in the infrared now obtains for the $x z, y z, 3 z^{2}-r^{2}$ bands, the situation that emerges is similar to the one $1,31,35$ found for the 1111-FeAs systems in early itinerant-based studies, though the present result places these bands much closer to the Mottness regime, as an examination of our results above shows. Thus, it is possible that small additional perturbations might tilt it into the incoherent regime. This analogy can now be used to directly predict that the superconduting state will most probably have in-plane $s_{ \pm}$pair symmetry. Further, depending on the extent of the $c$-axis corrugation of the three-dimensional (3D) Fermi surface, it will (or will not) have accidental $c$-axis nodes $\underline{32,36}$

It is thus quite remarkable that both, the nature and extent of orbital selectivity in hole- and electron-doped cases play a critical role in choosing the detailed set of conditions which determine the nature and symmetry of the superconducting state in each cases. The diversity of pairing states in Fe-based systems, by now appreciated to be a consequence of the MO nature of these systems, may thus be exposed in $\mathrm{Fe}$ oxychalcogenides if these can be driven superconducting by appropriate pressure, chemical and/or electrical doping. Whether this fascinating state of affairs unfolds in the future only time will tell.

\section{CONCLUSION}

To summarize, we have used LDA+DMFT for a minimally realistic five-band Hubbard model to perform a detailed study of a doped Mott insulator in the recently discovered $\mathrm{Fe}$ oxychalcogenide. In particular, considering $\mathrm{Na}_{2} \mathrm{Fe}_{2} \mathrm{OSe}_{2}$ as a suitable template, we have carefully analysed its paramagnetic Mott state, baring the orbital-selective Mott gap as an effect of multi-orbital Hubbard correlations. Much more interesting behavior is predicted when we consider electron- and hole 
doping of this state: Upon the latter, hole localization or incoherence persists because the chemical potential lies in a gap region with vanishing density of states, or in the low-energy pseudogap, which we ascribe to the blocking of coherent motion of doped holes due to a lattice orthogonality catastrophe induced by orbitalselective Mottness. On the other hand, in a remarkable difference, electron doping this Mott insulator leads to an orbital-selective low-energy quasi-coherent states coexisting with pseudogapped states, implying low-energy strongly correlated Fermi-liquid state with a small quasiparticle weight. These can be directly tested by a com- bination of future spectral and transport measurements. Finally, based upon the reconstructed Fermi surfaces implied by our results as well as upon the non-LFL or LFL nature of the metallic phases found here, we discuss the nature and symmetry of the possible unconventional superconducting states that one may find upon proper doping or pressurising the system. Such studies are called for, and should confirm or refute our predictions.

L.C. thanks the Brazilian funding agency CAPES (Proc. No. 002/2012) for financial support. Acknowledgment is also made to FAPEMAT/CNPq (Projet: $685524 / 2010)$.
1 S. Maiti and A.V. Chubukov, Phys. Rev. B bf 82,214515 (2010).

2 P.W. Anderson, The Theory of Superconductivity in the High-Tc Cuprates (Princeton University Press, Princeton, NJ, 1997).

3 S.C. Riggs, J.B. Kemper, Y. Jo, Z. Stegen, L. Balicas, G.S. Boebinger, F.F. Balakirev, A. Migliori, H. Chen, R.H. Liu, and X.H. Chen, Phys. Rev. B 79, 212510 (2009).

${ }^{4}$ R.W. Hill, C. Proust, L. Taillefer, P. Fournier, and R. L. Greene, Nature (London) 414, 711 (2001).

${ }^{5}$ C. Tarantini, M. Putti, A. Gurevich, Y. Shen, R.K. Singh, J.M. Rowell, N. Newman, D.C. Larbalestier, P. Cheng, Y. Jia, and H.-H. Wen, Phys. Rev. Lett. 104, 087002 (2010).

${ }^{6}$ Y. Mizuguchi, Tomioka F., Tsuda S., Yamaguchi T., and Y. Takano, J. Phys. Soc. Jpn. 78, 074712 (2009); M.H. Fang, J.H. Yang, F.F. Balakirev, Y. Kohama, J. Singleton, and B. Qian, Z.Q. Mao, H.D. Wang, and H.Q. Yuan, Phys. Rev. B 81, 020509 (2010).

7 Z.G. Chen, R.H. Yuan, T. Dong, G. Xu, Y.G. Shi, P. Zheng, J.L. Luo, J.G. Guo, X.L. Chen, and N.L. Wang, Phys. Rev. B 83, 220507(R) (2011); F. Chen, M. Xu, Q.Q. Ge, Y. Zhang, Z.R. Ye, L.X. Yang, J. Jiang, B.P. Xie, R.C. Che, M. Zhang, A.F. Wang, X.H. Chen, D.W. Shen, X.M. Xie, M.H. Jiang, J.P. Hu, and D.L. Feng, Phys. Rev. X 1, 021020 (2011).

8 P. Phillips, T.-P. Choy, and R.G. Leigh, Rep. Prog. Phys. 72, 036501 (2009).

9 S. Sachdev and B. Keimer, Physics Today 64, 29 (2011).

10 L. Craco, M.S. Laad, and S. Leoni, Phys. Rev. B 84, 224520 (2011).

11 L. Craco and S. Leoni, Europhys. Lett. 92, 67003 (2010).

12 J.-X. Zhu, R. Yu, H. Wang, L.L. Zhao, M.D. Jones, J. Dai, E. Abrahams, E. Morosan, M. Fang, and Q. Si, Phys. Rev. Lett. 104, 216405 (2010).

13 H. Kabbour, E. Janod, B. Corraze, M. Danot, C. Lee, M.H. Whangbo, and L. Cario, J. Am. Chem. Soc. 130, 8261 (2008).

14 N. Ni, S. Jia, Q. Huang, E. Climent-Pascual, and R. J. Cava, Phys. Rev. B 83, 224403 (2011).

15 J.B. He, D.M. Wang, H.L. Shi, H.X. Yang, J.Q. Li, and G.F. Chen, Phys. Rev. B 84, 205212 (2011).

16 F. Han, X. Wan, B. Shen, and H.-H. Wen, Physical Review B 86, 014411 (2012).

17 G. Baskaran, J. Phys. Soc. Jpn. 77, 113713 (2008); R. Yu, J.-X. Zhu, and Q. Si, Phys. Rev. Lett. 106, 186401 (2011).

18 Q. Si, Nature Phys. 5, 629 (2009); M.M. Qazilbash, J.J.
Hamlin, R.E. Baumbach, L. Zhang, D.J. Singh, M.B. Maple, D.N. Basov, Nature Phys. 5, 647 (2009).

19 T.-P. Choy and P. Phillips, Phys. Rev. Lett. 95, 196405 (2005).

20 G.S. Boebinger, Y. Ando, A. Passner, T. Kimura, M. Okuya, J. Shimoyama, K. Kishio, K. Tamasaku, N. Ichikawa, and S. Uchida, Phys. Rev. Lett. 77, 5417 (1996); see also, K. Semba and A. Matsuda, Phys. Rev. Lett. 86, 496 (2001).

21 J.W. Simonson, K. Post, C. Marques, G. Smith, O. Khatib, D.N. Basov, and M.C. Aronson, Phys. Rev. B 84, 165129 (2011).

22 Y.Shiomi, S.Ishiwata, Y.Taguchi, and Y.Tokura, Phys. Rev. B 84, 054519 (2011).

23 G. Kotliar, S.Y. Savrasov, K. Haule, V.S. Oudovenko, O. Parcollet, and C.A. Marianetti, Rev. Mod. Phys. 78, 865, (2006); K. Held, Advances in Physics 56, 829 (2007).

24 O.K. Andersen, Phys. Rev. B 12, 3060 (1975).

${ }^{25}$ L. Craco, Phys. Rev. B 77, 125122 (2008).

26 L. Craco, M. S. Laad, S. Leoni, and H. Rosner, Phys. Rev. B 78, 134511 (2008); M.S. Laad, L. Craco, S. Leoni, and H. Rosner, Phys. Rev. B. 79, 024515 (2009).

27 K. Haule, J. H. Shim, and G. Kotliar, Phys. Rev. Lett. 100, 226402 (2008); A. Kutepov, K. Haule, S.Y. Savrasov, and G. Kotliar, Phys. Rev. B 82, 045105 (2010); A. Liebsch and H. Ishida, Phys. Rev. B 82, 155106 (2010).

28 N. Barišić, D. Wu, M. Dressel, L.J. Li, G.H. Cao, and Z.A. Xu, Phys. Rev. B 82, 054518 (2010).

29 A. Liebsch, H. Ishida, and J. Merino, Phys. Rev. B 78, 165123 (2008).

30 M. Ferrero, P.S. Cornaglia, L. De Leo, O. Parcollet, G. Kotliar, and A. Georges, Phys. Rev. B 80, 064501 (2009).

31 I.I. Mazin, D.J. Singh, M.D. Johannes, and M.H. Du, Phys. Rev. Lett. 101, 057003 (2008).

32 M.S. Laad and L. Craco, Phys. Rev. Lett. 103, 017002 (2009).

33 R. Thomale, C. Platt, W. Hanke, and B.A. Bernevig, Phys. Rev. Lett. 106, 187003 (2011).

34 D.F. Agterberg, T.M. Rice, and M. Sigrist, Phys. Rev. Lett. 78, 3374 (1997).

35 V. Cvetkovic and Z. Tesanovic, Europhys. Letts. 85, 37002 (2009).

36 J.-Ph. Reid, M.A. Tanatar, X.G. Luo, H. Shakeripour, N. Doiron-Leyraud, N. Ni, S.L. Bud'ko, P.C. Canfield, R. Prozorov, and L. Taillefer, Phys. Rev. B 82, 064501 (2010). 